\title{
PERCEPCIÓN DE LA SALUD SEGÚN SEXO Y EDAD*
}

\section{HEALTH PERCEPTION BY GENDER AND AGE}

\author{
Marta Gil-Lacruz ${ }^{* *}$ \\ Ana Isabel Gil-Lacruz ${ }^{* * *}$
}

RESUMEN

Con este trabajo, pretendemos elaborar un diagnóstico de la salud de hombres y mujeres del barrio de Casablanca (Zaragoza). Aunque por lo general, la sociedad española se siente sana, las mujeres sufren más complicaciones de salud, bien sea, por diferencias biológicas o por características culturales. La aplicación empírica se ha llevado a cabo mediante una encuesta en un barrio zaragozano (1032 personas mayores de 14 años).

PALABRAS CLAVE: ZARAGOZA, ES * SALUD * SEXO * EDAD * DIAGNÓSTICO

\section{ABSTRACT}

The main goal of this paper is to elaborate a health diagnose is of men and women who live in the same Spanish neighborhood. Although in general Spanish people find themselves healthy, females present more health problems due to cultural differences or due to biological factors. The empirical estimation has been carried out with data drawn from a self-elaborated survey of 1032 people older than 14 years old.

KEYWORDS: ZARAGOZA, ES $*$ HEALTH $*$ GENDER $*$ AGE $*$ DIAGNOSIS

\section{INTRODUCCIÓN}

La disponibilidad de información fiable y oportuna sobre el estado de salud de la población es indispensable para la evalua- ción y planificación de los sistemas de salud. Tradicionalmente la salud se ha definido de forma negativa, pues se ha entendido como ausencia de enfermedad. Una de las principales implicaciones de esta concepción es que el
Este artículo es parte de una investigación mayor realizada en el Instituto de la Mujer bajo el proyecto I+D+I: Mujer y salud, aproximación psicosocial y comunitaria. Expediente 35/03. \footnotetext{
Universidad de Zaragoza, España.mglacruz@unizar.es

*** Universidad Autónoma de Madrid, España. ana.gil@uam.es.
} 
estado de salud se ha venido midiendo prácticamente con exclusividad mediante indicadores de morbilidad y mortalidad.

Una alternativa útil para la medición de la salud poblacional es la aplicación de encuestas en las cuales se pregunta a los individuos sobre su percepción del estado de salud. La principal ventaja de estas encuestas es que reflejan el concepto amplio de salud entendido como un estado de bienestar integral. Si hoy en día, nadie se cuestiona el hecho de que las necesidades relacionadas con una vida de calidad varían en función de la edad de la persona, de manera análoga tampoco se debería cuestionar la importancia que merecen las diferencias entre hombres $y$ mujeres.

Por esta razón, la principal aportación que realizamos con este trabajo es aproximar el estudio del estado de salud a una perspectiva de género. Sólo una vez que seamos conscientes de la dimensión del problema, estaremos en disposición de aportar orientaciones sobre el diseño de estrategias dirigidas a actuar y a cooperar en la mejora del estado de salud de hombres $y$ mujeres, $y$ por extensión, en la mejora del estado de salud de la sociedad en general. El principal objetivo de este trabajo es elaborar un diagnóstico sobre la salud de hombres y mujeres del barrio de Casablanca, en el que la percepción de la salud juega un papel principal.

Centrado el análisis empírico en nuestra comunidad, el motivo por el que seleccionamos el barrio de Casablanca es porque constituye una comunidad marcada por los contrastes $y$ las diferencias socio-demográficas. El universo de la presente investigación está compuesto por 1032 personas mayores de 14 años, empadronadas $^{1}$ en el barrio zaragozano de Casablanca (497 hombres y 535 mujeres).

Aunque la encuesta de salud nos proporciona una información valiosa y útil sobre las características del barrio y la interrelación de los diferentes tipos de variables, no deja de presentar ciertas limitaciones. Así, un desafío

1 El empadronamiento consiste en la acción de asentar o escribir a los ciudadanos en el padrón o libro de moradores de un pueblo, ya para la policía, el gobierno, el pago de tributos u otro fin análogo. añadido en el tratamiento de las encuestas ha estribado en priorizar tanto el impacto de las variables, como la interrelación de sus efectos. Por ejemplo, en el caso de la percepción del estado de salud, la variable edad puede condicionar considerablemente los resultados de los estudios que se centran en la conexión entre salud y sexo. De tal manera, las diferencias sexuales habría que contrastarlas con otras variables mediadoras, como los diferentes grupos de edad, puesto que con los años aumenta la heterogeneidad social.

Este trabajo se estructura de la siguiente manera. En la Sección 2 nos centramos en las investigaciones relativas a la percepción de la salud. En la Sección 3 detallamos las particularidades de la muestra con la que trabajamos. La Sección 4 y la Sección 5 se han destinado al marco contextual $y$ a los resultados inferenciales respectivamente. Finalizamos con la Sección 6 resumiendo las principales conclusiones obtenidas $y$ aportando diversas implicaciones políticas.

\section{LA PERCEPCIÓN DE LA SALUD}

Desde las ciencias comportamentales $y$ las encuestas de salud, la vertiente subjetiva del bienestar en la definición de salud está adquiriendo progresivamente una mayor relevancia científica (Diener y Larsen, 1993; Myers y Diener, 1995; Vanderzee, Buunk y Sanderman, 1995). Esto se debe a que para entender la definición de la salud, en términos positivos, necesitamos integrar aspectos psicosociales. La salud constituye un proceso dinámico en el que influyen distintos factores, tanto de carácter interno o personal como de carácter externo o ambiental. La salud no es una cuestión que se pueda acotar a su presencia o a su ausencia.

La Constitución de la Organización Mundial de la Salud, oms, define la salud como un estado de bienestar completo físico, mental $y$ social e insiste en que no puede circunscribirse a una ausencia de enfermedad o incapacidad. En este sentido, la mayoría de las definiciones actuales referidas a la salud son multidimensionales y abarcan tanto el funcionamiento objetivo como el bienestar percibido, articulando a su vez la calidad de vida. 
Con respecto a la realidad de nuestro entorno, constatamos que la población española se siente fundamentalmente sana. Para más de dos tercios de los consultados durante el 2002, su estado de salud ha sido bueno (Cuesta, 2003). Este dato es corroborado por la encuesta de FOESSA, en la que más de la mitad de la personas jóvenes (18-21 años) se consideran totalmente sanas. No obstante, dicho porcentaje se reduce considerablemente con la edad, ya que sólo un $11 \%$ de las personas mayores de 60 años se perciben totalmente sanas (Cais y Castilla, 1995).

Pero en esta tendencia, como viene siendo habitual, encontramos de nuevo diferencias preceptuales en función de las distintas categorías sociales básicas: sexo, edad y estado civil. Las mujeres se sienten peor que los hombres, $y$ consecuentemente, consideran que su estado de salud es más deficiente. Aunque estas pautas ratifican los trabajos científicos llevados a cabo (Braverman y Tarimo, 2002; Carpenter, 2000; Stevens, Seid, Mistry y Halfon, 2006), se deberían contrastar hipótesis alternativas en las que por ejemplo la mujer acepte mejor el etiquetaje de la enfermedad y no le incomode tanto el relato de sus dolencias (De Miguel, 1998; García y Soler, 2002; Gobierno de Navarra, 1991).

Ante edades semejantes, las mujeres no sólo valoran su salud peor que los varones, sino que además presentan más achaques sobre todo a partir de los 45 años. Esta diferencia se amplía en el grupo de los mayores de 65 años, debido básicamente a que comprende más mujeres que varones al ser su supervivencia objetiva es más alta (Encuesta Española de Salud, 1995). Dada esta situación, una de las hipótesis que justifican que la morbilidad femenina sea mayor, es que las mujeres sufran más molestias, estados de ansiedad y sintomatologías (De Miguel, 1997). La morbilidad objetiva de las mujeres se traduce en la consideración subjetiva de una salud más deficiente.

Las variables culturales también inciden en esta percepción. En nuestros trabajos es necesario trascender el nivel de análisis puramente individual y biológico y situar a la comunidad como el contexto en el que la salud es simultáneamente objeto de influencia y motor social de cambio (Sofaer and Firminger, 2005;
Thorson and Johansson, 2004). De ahí se infiere la importancia de considerar en el estudio de la salud el efecto de las variables culturales $y$ de los factores comunitarios en los que el individuo se socializa. Para algunas mujeres el cuerpo es percibido como un lugar de esfuerzo y sufrimiento, relacionado especialmente con los mandatos de género en cuanto a la sexualidad, la estética y la procreación; mientras que para los varones, el cuerpo está relacionado con la dureza, la fuerza y la violencia. En la conceptualización del binomio salud-enfermedad intervienen procesos de construcción simbólica de los cuerpos sexuados que afectan a la salud. Estas construcciones influyen en cómo las mujeres $y$ hombres interpretan $y$ viven los comportamientos que resultan de valoraciones diferenciales en la afirmación de las identidades de género, como por ejemplo la reproducción. Las diferentes experiencias e interpretaciones darán como resultado distintas maneras de vivir y entender la salud y la enfermedad (Hellinger and Encinosa, 2004).

Desde una perspectiva clínica, disponemos de una larga lista de malestares asociados a situaciones concretas, (ej. la menopausia), sin embargo, desde la práctica médica se ha olvidado que el sentir de las mujeres no tiene por qué responder necesariamente a esta descripción $y$ que, muchas veces, la vivencia es perfilada por la descripción de este cuadro más que a la inversa. Todo evento biológico va acompañado de una experiencia subjetiva que transforma aquello que se llama "enfermedad" en un "padecimiento", diferencia que ha sido señalada claramente desde la antropología (Conrad y Schneider, 1980; Menéndez, 1990).

\section{METODOLOGÍA}

El universo de la presente investigación se compone por 6514 personas mayores de 14 años $y$ empadronadas en el barrio zaragozano de Casablanca (3 136 hombres y 3378 mujeres). Casablanca constituye una comunidad marcada por los contrastes $y$ las diferencias de composición socio-demográfica (Federación de Asociaciones de Vecinos, 1981; Gobierno de Aragón, 1998). 
Las diferencias de estratificación social se proyectan claramente en tres zonas tradicionales de residencia (Viñedo Viejo: núcleo originario y precarizado; Fuentes Claras: urbanizaciones de nivel socioeconómico elevado y Las Nieves: clase media y obrera).

En función de los datos disponibles del Padrón Municipal, la población objeto de estudio y la muestra se dividió en diversos grupos que denominamos estratos, obteniéndose a continuación una muestra proporcional representativa de cada estrato (García Ferrando, 1992). Los dos criterios básicos de estratificación de la muestra fueron: la edad (joven de 15 a 29 años, adulta de 30 a 59 y adulto mayor a partir de los 60 años) y el sexo (hombre y mujer) (Ver Cuadro 1).

Como se trabaja con una población finita (tiene menos de 100000 elementos y $\mathrm{N}<20 \mathrm{n}$, la población contiene menos de 20 veces el tamaño muestral; Mateo Rivas y García, 1989), para que la muestra sea representativa a un nivel de confianza de $95,5 \%$ y con un margen de error de \pm 3 (en el caso más desfavorable p/q=50/50 García Ferrando, 1992), se han contemplado 938 encuestas individuales (más un 10\% de reserva: 94 encuestas).

En cuanto a los indicadores que recoge la encuesta destacamos los siguientes por su implicación en el presente trabajo:

1. Características sociodemográficas: El primer test de la encuesta consta de indicadores de estratificación social (ej. situación laboral, nivel de estudios, ocupación, ingresos anuales, etc.), territoriales (lugar de nacimiento, lugar de residencia, años de residencia, etc.) y familiares (estado civil, número de miembros que residen en la unidad familiar, parentesco, etc.).

2. Percepción de la salud: Se utilizaron dos instrumentos de medida: uno centrado en

\section{CUADRO 1}

UNIVERSO Y MUESTRA DE LA POBLACIÓN DE CASABLANCA

\begin{tabular}{|c|c|c|c|c|c|c|}
\hline \multirow{4}{*}{$\begin{array}{l}\text { INTERVALOS EN FUNCIÓN } \\
\text { DE LA EDAD } \backslash S E X O\end{array}$} & \multicolumn{4}{|c|}{ POBLACIÓN DE CASABLANCA (N=6 514) } & \multicolumn{2}{|c|}{ MUESTRA $(\mathrm{n}=938)$} \\
\hline & \multicolumn{2}{|c|}{ HOMBRES } & \multicolumn{2}{|c|}{ MUJERES } & \multirow{3}{*}{$\begin{array}{c}\text { HOMBRES } \\
\mathrm{N} \\
\text { CUESTIONARIO }\end{array}$} & \multirow{3}{*}{$\begin{array}{c}\text { MUJERES } \\
\mathrm{N} \\
\text { CUESTIONARIO }\end{array}$} \\
\hline & FR & FR & FR & FR & & \\
\hline & ABSOLUTA & RELATIVA & ABSOLUTA & RELATIVA & & \\
\hline De 15 a 19 años & 410 & 6,29 & 412 & 6,32 & 59 & 59 \\
\hline De 20 a 24 años & 371 & 5,70 & 359 & 5,51 & 53 & 52 \\
\hline De 25 a 29 años & 312 & 4,79 & 265 & 4,07 & 45 & 38 \\
\hline TOTAL JUVENTUD & 1093 & 16,78 & 1036 & 15,90 & 157 & 149 \\
\hline De 30 a 34 años & 225 & 3,45 & 231 & 3,55 & 32 & 33 \\
\hline De 35 a 39 años & 223 & 3,42 & 252 & 3,87 & 32 & 36 \\
\hline De 40 a 44 años & 275 & 4,22 & 327 & 5,02 & 40 & 47 \\
\hline De 45 a 49 años & 289 & 4,44 & 319 & 4,90 & 42 & 46 \\
\hline De 50 a 54 años & 260 & 3,99 & 231 & 3,55 & 37 & 33 \\
\hline De 55 a 59 años & 224 & 3,44 & 247 & 3,79 & 32 & 36 \\
\hline TOTAL ADULTOS & 1496 & 22,97 & 1607 & 24,67 & 215 & 231 \\
\hline De 60 a 64 años & 196 & 3,01 & 196 & 3,01 & 28 & 28 \\
\hline De 65 a 69 años & 137 & 2,10 & 199 & 3,05 & 20 & 29 \\
\hline De 70 a 74 años & 102 & 1,57 & 110 & 1,69 & 15 & 16 \\
\hline De 75 a 79 años & 55 & 0,84 & 101 & 1,55 & 8 & 15 \\
\hline$>$ de 80 años & 57 & 0,88 & 129 & 1,98 & 8 & 19 \\
\hline TOTAL TERCERA EDAD & 547 & 8,40 & 735 & 11,28 & 79 & 106 \\
\hline TOTALES & 3136 & 48,14 & 3378 & 51,86 & 452 & 486 \\
\hline
\end{tabular}

Fuente: Elaboración propia. 
morbilidad y el otro en la percepción general de salud. En cuanto al primer instrumento, como en la Encuesta de Salud de Zaragoza (1991), hemos definido y clasificado: a) La morbilidad percibida aguda como la dolencia o enfermedad que ha afectado al entrevistado en las dos últimas semanas, excluidos los síntomas de procesos crónicos; b) La morbilidad percibida crónica como la enfermedad que se ha manifestado hace más de tres meses de forma permanente o con cierta frecuencia; c) Las respuestas aportadas en relación con el diagnóstico realizado por el servicio de salud se han codificado mediante el sistema WONCA; d) El grado de incapacitación.
El segundo instrumento utilizado en relación a la percepción de salud es el CPS-81 (Davis y Ware, 1981), que evalúa las siguientes dimensiones: salud anterior, salud actual, previsión de la salud en un futuro, resistencia frente a la enfermedad, tendencia a aceptar la enfermedad como una parte de la propia vida y preocupación por el estado de salud. A través de los 30 ítems que compone la escala se puede obtener un índice general de salud (27 ítems) y un índice breve de salud (3 ítems). La consistencia interna del instrumento es elevada (el coeficiente $\alpha$ del total de la escala es 0,8614 , en un intervalo de medición que oscila entre el 0 y el 1). En el cuadro cuadro 2 se expone información relativa a la

CUADRO 2

ANÁLISIS ESTADÍSTICO DE LA CONSISTENCIA INTERNA DE LOS ÍTEMS

\begin{tabular}{|c|c|c|c|c|c|c|}
\hline ÍTEM & NOMBRE & MEDIA & DESVIACIÓN & $\begin{array}{c}r \\
\text { CORRELACIÓN DE LA } \\
\text { ESCALA EXCLUYENDO EL } \\
\text { ÍTEM } \\
\end{array}$ & $\begin{array}{c}\mathrm{R} \\
\text { COEFICIENTE DE } \\
\text { CORRELACIÓN MÚLTIPLE AL } \\
\text { CUADRADO } \\
\end{array}$ & ÍNDICE A \\
\hline 1 & PERSA01 & 2,5271 & 0,7923 & 0,6385 & 0,5034 & 0,8513 \\
\hline 2 & PERSA02 & 3,0039 & 1,0197 & 0,5537 & 0,4479 & 0,8522 \\
\hline 3 & PERSA03 & 2,7800 & 1,0409 & 0,4837 & 0,3602 & 0,8543 \\
\hline 4 & PERSA04 & 3,6240 & 0,8530 & 0,5342 & 0,3921 & 0,8535 \\
\hline 5 & PERSA05 & 3,9089 & 0,8560 & 0,4504 & 0,3484 & 0,8556 \\
\hline 6 & PERSA06 & 3,0223 & 0,9500 & 0,5679 & 0,5300 & 0,8521 \\
\hline 7 & PERSA07 & 3,5136 & 0,9445 & 0,3135 & 0,2988 & 0,8593 \\
\hline 8 & PERSA08 & 2,5407 & 1,0122 & 0,1575 & 0,2783 & 0,8640 \\
\hline 9 & PERSA09 & 2,9612 & 0,8833 & 0,2440 & 0,2893 & 0,8609 \\
\hline 10 & PERSA10 & 3,3227 & 1,0402 & 0,6244 & 0,5336 & 0,8498 \\
\hline 11 & PERSA11 & 2,8401 & 0,7821 & 0,1788 & 0,2600 & 0,8621 \\
\hline 12 & PERSA12 & 3,9157 & 1,2204 & 0,3230 & 0,2626 & 0,8601 \\
\hline 13 & PERSA13 & 2,9457 & 1,0864 & 0,4530 & 0,3614 & 0,8553 \\
\hline 14 & PERSA14 & 3,1996 & 0,9138 & 0,1867 & 0,2386 & 0,8626 \\
\hline 15 & PERSA15 & 3,7994 & 0,6705 & 0,2390 & 0,1582 & 0,8605 \\
\hline 16 & PERSA16 & 3,3101 & 1,0881 & 0,3703 & 0,2174 & 0,8579 \\
\hline 17 & PERSA17 & 3,0107 & 0,7994 & 0,3188 & 0,3161 & 0,8589 \\
\hline 18 & PERSA18 & 3,5320 & 0,9513 & 0,3418 & 0,3318 & 0,8585 \\
\hline 19 & PERSA19 & 3,7481 & 0,9682 & 0,5495 & 0,4019 & 0,8525 \\
\hline 20 & PERSA20 & 2,8973 & 0,9181 & 0,1011 & 0,2645 & 0,8648 \\
\hline 21 & PERSA21 & 3,2306 & 0,9765 & 0,6950 & 0,5642 & 0,8481 \\
\hline 22 & PERSA22 & 3,9564 & 0,6774 & 0,1931 & 0,1492 & 0,8614 \\
\hline 23 & PERSA23 & 2,5203 & 0,9351 & 0,2084 & 0,2987 & 0,8621 \\
\hline 24 & PERSA24 & 2,4225 & 0,8808 & 0,1012 & 0,1224 & 0,8645 \\
\hline 25 & PERSA25 & 3,3653 & 1,0326 & 0,5749 & 0,4478 & 0,8515 \\
\hline 26 & PERSA26 & 3,9196 & 0,9376 & 0,5040 & 0,4272 & 0,8539 \\
\hline 27 & PERSA27 & 4,1047 & 0,7228 & 0,3193 & 0,2312 & 0,8589 \\
\hline 28 & PERSA28 & 4,0136 & 0,7499 & 0,5147 & 0,4402 & 0,8546 \\
\hline 29 & PERSA29 & 2,8886 & 0,9145 & 0,5590 & 0,5053 & 0,8525 \\
\hline
\end{tabular}

Fuente: Elaboración propia. 
aportación de cada uno de los ítems a la consistencia interna de la escala.

\section{MARCO CONTEXTUAL}

Si por algo se caracteriza el barrio de Casablanca es por su diversidad $y$ heterogeneidad social. De ser una zona de regadíos y de recreo (por la presencia del Canal Imperial de Aragón) a finales del siglo XIX, evolucionó a mitad del siglo Xx a zona residencial periférica de una ciudad polo de desarrollo industrial (Zaragoza). Desde 1960 y hasta la fecha, la estructura poblacional $y$ las tradicionales tareas agrarias e industriales, se han transformado en actividades económicas y socio-profesionales urbanas.

Hoy en día, constituye un vecindario marcado por los contrastes y las diferencias de composición sociodemográfica, que en parte se justifican por la aceleración de los cambios pasados. El núcleo tradicional de calles estrechas y viviendas de ladrillo de tres, cuatro plantas sin ascensor, convive con el actual proceso de especulación y los chalets verjados y unifamiliares. La trayectoria histórica y social del barrio puede sernos de gran utilidad para entender los procesos vecinales de otras comunidades.

A continuación detallamos unos primeros estadísticos descriptivos que nos aportan una primera visión de la esencia del estudio en la comunidad objeto de estudio. Empezaremos con modelos descriptivos y tablas de contingencia con el fin de mostrar las características generales del barrio, incidiendo especialmente en los rasgos particulares de sus hombres $y$ mujeres. A partir de aquí, desarrollaremos medidas de asociación $y$ de inferencia que nos permitan generalizar los resultados al universo de la investigación y establecer relaciones estadísticamente significativas entre las variables.

\subsection{CARACTERÍSTICAS SOCIODEMOGRÁFICAS}

Se han entrevistado a 497 hombres y a 535 mujeres del barrio. El número de encuestas supone una proporción de 48,2\% y 51,8\% respectivamente, que coincide con los porcentajes obtenidos en el universo de la investigación.
El promedio de edad de los encuestados es de 41 años, $y$ a medida que aumenta la edad, mayor es el número de mujeres. También destacaremos que la distribución de sexos y de edades difiere en función de la zona de residencia, ya que es en Viñedo Viejo (núcleo originario del barrio) donde encontramos una mayor presencia de mujeres mayores. Estas diferencias se reflejan en el estado civil, ya que de nuevo, en esta zona encontramos una mayor proporción de matrimonios y personas viudas. En cambio, los solteros y solteras se asientan en las zonas de reciente colonización (Fuentes Claras). Las unidades familiares de Casablanca se componen generalmente de tres o cuatro miembros (media $=3,85$, moda $=4 \mathrm{y}$ desviación típica $=1,48$ ).

En la muestra seleccionada trabaja un $36,9 \%$ de los encuestados. Sin embargo, esta cifra oculta importantes diferencias territoriales. Mientras que en Fuentes Claras este porcentaje es de 48,1\%, en Viñedo Viejo es de 35\%. En Fuentes Claras, la proporción de personas que se dedican a tareas del hogar (11,5\%), también es sensiblemente inferior que en Viñedo Viejo (24,9\%).

Tres de cada cuatro personas entrevistadas $(75,8 \%)$ ha cursado la enseñanza elemental y/o secundaria. Un $5,3 \%$ de la muestra no ha estudiado $y$ un $18,9 \%$ ha alcanzado una titulación universitaria. Parte de esta diversidad se proyecta en el nivel de ingresos: un 47,8\% de los encuestados obtiene unos ingresos anuales inferiores a 12000 euros. Sólo un 15,1\% supera los 24000 euros $^{2}$.

En cuanto al tiempo de residencia de los vecinos en el barrio observamos los diferentes procesos migratorios: más de la mitad de los encuestados de Fuentes Claras (59,6\%) residen en la comunidad desde hace menos de 9 años. Por el contrario, en Viñedo Viejo una cifra similar

En España, según estadísticas calculadas por el Eurostat, el salario mínimo interprofesional gira en torno a los 513 euros, elevado hasta 600 euros mensuales si se tiene en cuenta las dos pagas extraordinarias anuales que en otros países no se abonan. Sobre esa base, el sueldo básico en España resulta 42 euros inferior a la media de los 18 países mencionados: 641 euros (Finanzas.com, 17/04/2002). 
$(59,1 \%)$ corresponde a los que llevan viviendo en la zona más de 20 años.

\subsection{PERCEPCIÓN DE LA SALUD}

Al recurrir al cuestionario de percepción de la salud, CPS, de Davis y Ware (1981), observamos claramente que las mujeres de Casablanca se sienten peor que los hombres. Consideran que, tanto en pasado como en el presente, tienen peor salud que sus compañeros, aunque sin embargo son optimistas con respecto a su futuro. En general, a las mujeres los temas de salud les preocupan más y están más pendientes de su sintomatología que sus compañeros. (Ver Cuadro 3).

CUADRO 3

PERCEPCIÓN DE LA SALUD EN FUNCIÓN DEL SEXO

\begin{tabular}{cccccccc}
\hline $\begin{array}{c}\text { SEXO DEL } \\
\text { ENCUESTADO } \\
\text { (MEDIAS) }\end{array}$ & $\begin{array}{c}\text { SALUD } \\
\text { ACTUAL }\end{array}$ & $\begin{array}{c}\text { SALUD } \\
\text { PASADA }\end{array}$ & $\begin{array}{c}\text { EXPECTATIVAS } \\
\text { DE } \\
\text { SALUD }\end{array}$ & $\begin{array}{c}\text { RESISTENCIA } \\
\text { A } \\
\text { ENFERMEDAD }\end{array}$ & $\begin{array}{c}\text { PREOCUPACIÓN } \\
\text { POR LA } \\
\text { SALUD }\end{array}$ & $\begin{array}{c}\text { ORIENTACIÓN } \\
\text { A LA } \\
\text { ENFERMEDAD }\end{array}$ & $\begin{array}{c}\text { ÍNDICE } \\
\text { GENERAL DE } \\
\text { SALUD }\end{array}$ \\
\hline $\begin{array}{c}\text { Hombre } \\
\text { Mujer }\end{array}$ & 30,01 & 11,25 & 13,82 & 14,94 & 14,02 & 6,19 & 76,22 \\
TOTAL & 28,87 & 10,75 & 13,86 & 14,62 & 14,40 & 6,34 & 74,56 \\
& 29,42 & 10,99 & 13,84 & 14,77 & 14,22 & 6,27 & 75,36 \\
\hline
\end{tabular}

Fuente: Elaboración propia.

Sólo un 20,4\% de la muestra manifiesta no haber sufrido ninguna dolencia aguda 0 crónica, mientras que el $42,2 \%$ de los residentes han compartido sintomatologías agudas $y$ crónicas. El 37,2\% de la población presenta sólo un tipo de malestar en esta doble clasificación.

Cuando preguntamos a los encuestados sobre las complicaciones de salud padecidas durante los últimos 15 días, estas se centran en: dolores de huesos, columna o articulaciones (26,5\% del total de la muestra), dolor de cabeza $(20,4 \%)$, tos, catarro, gripe $(18,8 \%)$, problemas de garganta $(12,7 \%)$, problemas de nervios o depresión (11\%), dificultades para dormir (10,3\%) y cansancio sin razón aparente (7,8\%). Llama la atención el hecho de que las personas que sufren problemas de salud agudos, relatan un promedio de 1,7 dolencias. Muchas de estas dolencias pueden deberse a los estilos de vida (por su carácter psicosomático) y al momento en el que se realiza la entrevista (invierno).

En cuanto a la morbilidad crónica destacamos varios grupos de trastornos. En primer lugar se encuentra el reumatismo, la artrosis, la artritis, la ciática, el lumbago, el dolor de espalda (20,9\% sobre el total de la muestra), la presión arterial alta (7\%) y las alergias (5,8\%). En un segundo grupo, destacarían las dolencias de nuevo con una clara raíz psicosomática: el dolor de cabeza, la jaqueca, la migraña $(8,9 \%)$, los problemas de nervios, la depresión y los trastornos mentales (8,3\%), y las dolencias de estómago (5,2\%). Entre las dolencias no psicosomáticas el colesterol ocupa un lugar destacado $(6,9 \%)$.

Casi la mitad de los encuestados (47\%) presentaba en el momento de realizar la encuesta, diagnósticos sanitarios sobre su estado de salud. Sin embargo, este dato hay que interpretarlo con cautela puesto que los trastornos mentales pueden pasar desapercibidos. Mientras que un $8,3 \%$ de los encuestados plantean que sufren problemas de nervios, depresión o trastornos mentales, sólo el 1,7\% de los mismos, obtuvo como primer diagnóstico médico dicha catalogación.

\section{RESULTADOS INFERENCIALES}

Los análisis de frecuencias planteados anteriormente suponen un primer acercamiento a la descripción de las características de la muestra. No obstante, resulta necesario profundizar en la dispersión que presentan las variables y su cambiabilidad en función de determinadas características. Con este fin, hemos utilizado el modelo simple de comportamiento 
ANOVA (una sola variable de intervalo y una 0 varias variables nominales, Serra, 1994). Se ha elegido como nivel mínimo de significación estadístico $\alpha=0,05$ para rechazar la hipótesis de que las medias de las poblaciones son iguales 0 no difieren en términos de la variable independiente, $y$ por tanto, la dispersión se debe simplemente al azar (Serret, 1995).

Para entender el grado de interdependencia entre todas las variables relativas a la percepción de la salud llevamos a cabo un análisis discriminante sobre el índice general de salud. La morbilidad crónica, diagnosticada y aguda, contribuiría en primer lugar a la predicción de esta valoración. En un segundo orden de importancia encontraríamos ciertos factores objetivos como el número de dolencias diagnosticadas por un profesional de la salud, el número de consultas médicas realizadas en los últimos 15 días y el grado de incapacidad que han ocasionado dichas molestias. En tercer lugar, la inactividad es una variable que se relaciona directamente con un peor pronóstico de la evaluación de la propia salud (Gil Lacruz, 2000).

Como, para la mayoría de las variables, se trabaja con el cruce de dos posibles categorías de la variable sexo (hombre y mujer), no se ha considerado necesario, establecer procedimientos posteriores que señalen las diferencias estadísticamente significativas de las diversas respuestas de la población (Canovos, 1988).

\subsection{PERCEPCIÓN DE LA SALUD}

Se aprecian diferencias significativas en todos los factores que miden dolencias percibidas $y$ diagnosticadas, independientemente del tiempo que consideremos como referencia. Mientras las mujeres han padecido un promedio de 1,97 síntomas en los 15 días previos a la entrevista, la media masculina se reduce a 1,41 . En cuanto a morbilidad crónica los hombres presentan menos complicaciones de salud $(0,96)$ que las mujeres $(1,59)$. Estas tendencias se observan en la respuesta de los sistemas sanitarios. Las dolencias de las mujeres del barrio han sido diagnosticadas con más frecuencia (Ver Cuadro 4).

Sin embargo, un resultado que llama la atención, porque puede parecer contradictorio con las tendencias anteriores, estriba en que los hombres sufren menos complicaciones de salud pero estas les incapacitan más. Así, han tenido que, por un lado, reducir su tiempo libre y el destinado a su actividad principal $y$, por otro, permanecer en la cama más tiempo que las mujeres del barrio.

Durante las dos semanas anteriores a la entrevista, tanto hombres como mujeres coinciden en que sus principales molestias de salud han tenido que ver con dolores de huesos, columna o articulaciones; tos, catarro o gripe o dolores de cabeza y/o garganta. Pero, en determinadas complicaciones de salud, las diferencias son considerables. Por ejemplo, el $14,2 \%$ de las mujeres encuestadas han padecido

CUADRO 4

ANÁLISIS DE VARIANZA DE MORBILIDAD E INCAPACIDAD

\begin{tabular}{lccccc}
\hline VARIABLES & $\begin{array}{c}\text { SUMA DE } \\
\text { CUADRADOS }\end{array}$ & $\mathrm{F}$ & SIG. & HOMBRE & MUJER \\
\hline Suma ítems morbilidad aguda & 77,858 & 18,626 & 0,000 & 1,42 & 1,97 \\
Suma items morbilidad crónica & 99,399 & 32,813 & 0,000 & 0,97 & 1,59 \\
Suma items ambas morbilidades & 17,609 & 31,254 & 0,000 & 1,08 & 1,34 \\
Suma diagnósticos WONCA & 25,3716 & 36,585 & 0,000 & 0,50 & 0,82 \\
Grado de incapacitación & 20,8415 & 18,781 & 0,000 & 0,36 & 0,65 \\
Incapacidad tiempo libre & 1,065 & 6,115 & 0,014 & 1,81 & 1,74 \\
Incapacidad actividad principal & 1,498 & 9,055 & 0,003 & 1,84 & 1,75 \\
Incapacidad autonomía & 0,210 & 3,753 & 0,053 & 1,96 & 1,93 \\
Incapacidad permanecer en cama & 0,743 & 6,027 & 0,014 & 1,89 & 1,83 \\
\hline
\end{tabular}

Fuente: Elaboración propia 
problemas de nervios o depresión $(7,6 \%$ en el caso de los hombres).

Este problema puede encubrir otras molestias de carácter psicosomático, que también son más frecuentes en las mujeres, como son las dificultades para dormir, molestias de estómago, de cabeza y/o mareos (Ver Cuadro 5).

\section{CUADRO 5}

SINTOMATOLOGÍA DE LA MORBILIDAD AGUDA EN FUNCIÓN DEL SEXO (\%)

\begin{tabular}{|c|c|c|c|c|c|}
\hline SINTOMATOLOGÍA & HOMBRES & MUJERES & SINTOMATOLOGÍA & HOMBRES & MUJERES \\
\hline Dolor de huesos, columna... & 21,1 & 31,4 & $\begin{array}{l}\text { Problemas con los } \\
\text { dientes... }\end{array}$ & 7,8 & 7,5 \\
\hline $\begin{array}{l}\text { Problemas de nervios o } \\
\text { depresión }\end{array}$ & 7,6 & 14,2 & $\begin{array}{l}\text { Problemas periodo o } \\
\text { menopausia }\end{array}$ & 0.0 & 7,3 \\
\hline Mareos o vahídos & 4,0 & 7,3 & Tobillos hinchados & 1,4 & 4,9 \\
\hline Fiebre & 3,2 & 4,5 & $\begin{array}{l}\text { Ahogo, dificultad para } \\
\text { respirar }\end{array}$ & 3,4 & 3,6 \\
\hline Problemas de garganta & 10,3 & 15,0 & $\begin{array}{l}\text { Cansancio sin razón } \\
\text { aparente }\end{array}$ & 5,6 & 9,7 \\
\hline Dolor en el pecho & 4,2 & 2,4 & Ronchas, picor & 2,4 & 3,0 \\
\hline Dolor de cabeza & 16,9 & 23,7 & Molestias de hígado & 0,4 & 1,5 \\
\hline Contusiones, lesión o herida & 6,2 & 2,4 & $\begin{array}{l}\text { Diarreas o problemas } \\
\text { intestinales }\end{array}$ & 3,0 & 3,2 \\
\hline Tos, catarro o gripe & 17,9 & 19,6 & Problemas de estómago & 5,8 & 7,7 \\
\hline Dolor de oídos & 1,6 & 6,2 & Dificultad para dormir & 8,9 & 11,6 \\
\hline Molestias de riñón o urinarias & 3,0 & 5,4 & Ojos (conjuntivitis) & 4,6 & 4,3 \\
\hline Presión arterial alta & 4,2 & 4,3 & Otros & 3,8 & 3,9 \\
\hline
\end{tabular}

Fuente: Elaboración propia.

En este sentido, $y$ de manera especialmente grave, sería la sintomatología relativa al cansancio: una de cada diez mujeres encuestadas se sentía cansada sin razón aparente (en los hombres esta proporción descendía al 5,6\% en el momento de realizar la entrevista). Como contrapunto, las contusiones, lesiones o heridas son más frecuentes en los hombres (mujeres: $2,4 \%$ del total de las encuestadas, hombres: 6,2\%) (Ver Cuadro 6).

\section{CUADRO 6}

SINTOMATOLOGÍA DE LA MORBILIDAD CRÓNICA (\%)

\begin{tabular}{|c|c|c|c|c|c|}
\hline & HOMBRES & MUJERES & & HOMBRES & MUJERES \\
\hline Colesterol & 6,2 & 7,5 & Anemia & 0,0 & 4,5 \\
\hline $\begin{array}{l}\text { Reumatismo, artrosis, dolor } \\
\text { espalda }\end{array}$ & 15,3 & 26,2 & $\begin{array}{l}\text { Problemas de hígado, vesícula } \\
\text { biliar }\end{array}$ & 1,2 & 2,8 \\
\hline Problemas de boca o encías & 3,4 & 6,0 & Problemas de corazón & 2,2 & 3,9 \\
\hline Hemorroides o almorranas & 5,0 & 3,4 & $\begin{array}{l}\text { Problemas de periodo, } \\
\text { menopausia }\end{array}$ & 0,0 & 8,2 \\
\hline Várices & 1,8 & 6,9 & $\begin{array}{l}\text { Dolor de cabeza, jaqueca, } \\
\text { migraña }\end{array}$ & 3,8 & 13,6 \\
\hline Gota, ácido úrico & 2,2 & 0,9 & Infecciones de orina, cistitis & 1,4 & 3,0 \\
\hline $\begin{array}{l}\text { Problemas persistentes con } \\
\text { los pies }\end{array}$ & 2,4 & 5,0 & Hernia & 1,4 & 1,3 \\
\hline Presión arterial alta & 6,8 & 7,1 & Dolores de estómago & 5,6 & 4,9 \\
\hline Tumor & 0,2 & 0,9 & $\begin{array}{l}\text { Problemas persistentes en } \\
\text { la piel }\end{array}$ & 4,4 & 5,6 \\
\hline Piedras en el riñón & 1,6 & 1,3 & Estreñimiento & 2,2 & 7,3 \\
\hline Algún tipo de defecto físico & 1,4 & 0,6 & Bronquitis o asma & 2,4 & 2,1 \\
\hline Depresión, trastorno mental & 4,4 & 12,0 & Problemas con los ojos & 6,2 & 4,1 \\
\hline Alergias & 6,2 & 5,4 & Otros & 7,0 & 10,7 \\
\hline Diabetes, azúcar en la sangre & 1,4 & 3,6 & & & \\
\hline
\end{tabular}

Fuente: Elaboración propia. 
Estos resultados son coherentes con los datos obtenidos en cuanto a la morbilidad percibida crónica. De nuevo, el reumatismo, la artrosis o artritis, la ciática, el lumbago o el dolor de espalda constituyen el conjunto de síntomas que se ha manifestado con más frecuencia en los encuestados.

Al intentar priorizar los siguientes indicadores, observamos que hombres y mujeres difieren en sus molestias crónicas. En los hombres predominan los problemas de presión arterial alta (6,8\% de los encuestados), colesterol $(6,2 \%)$ y alergias $(6,2 \%)$. En las mujeres volvemos a encontrar trastornos recurrentes $y$ similares a los que se sufrían de manera aguda: los dolores de cabeza $(13,6 \%)$ y los nervios o depresión (12,09\%) (Ver Cuadro 7).

Por otro lado, los datos apuntan a que $2,6 \%$ de las mujeres encuestadas padecen trastornos mentales. Las enfermedades relacionadas con los sistemas: osteomuscular, circulatorio y endocrino constituyen los diagnósticos más frecuentes cuando los encuestados y encuestadas acuden a las consultas médicas.

Con base a la evidencia empírica, las mujeres presentan una mayor vulnerabilidad en cuanto a la morbilidad aguda, crónica y diagnosticada en su percepción de la salud. Las diferencias son estadísticamente significativas en cuanto a la salud actual, pasada, la valoración de la resistencia a la enfermedad, la preocupación por la salud y el índice general de salud que integra al total de los ítems.

Los resultados presentan además una clara adscripción geográfica. Los encuestados del núcleo originario (Viñedo Viejo) exponen una valoración más negativa de su estado de salud anterior, actual y futura. La interpretación de estos resultados puede apuntar a varias hipótesis relacionadas con el sexo y edad de sus residentes. Así como primera hipótesis planteamos el envejecimiento poblacional de estas zonas pues es en este barrio donde se concentra una mayor proporción de personas mayores. Como segunda hipótesis, que se puede considerar complementaria a la primera, planteamos que en las zonas más antiguas del barrio residen aquellos individuos que padecen una mayor vulnerabilidad social en cuanto al nivel de instrucción, ingresos o situación laboral de sus habitantes. Ante esta situación no es de extrañar que las mujeres presenten una valoración más negativa

\section{CUADRO 7}

DIAGNÓSTICO DE LAS DOLENCIAS EN FUNCIÓN DEL SEXO

\begin{tabular}{|c|c|c|c|c|c|c|}
\hline \multirow{2}{*}{$\begin{array}{l}\text { DIAGNÓSTICO MEDICO SEGÚN } \\
\text { CLASIFICACIÓN WONCA } \\
\end{array}$} & \multicolumn{4}{|c|}{ SEXO DEL ENCUESTADO } & \multirow{2}{*}{\multicolumn{2}{|c|}{ TOTAL }} \\
\hline & \multicolumn{2}{|c|}{ HOMBRE } & \multicolumn{2}{|c|}{ MUJER } & & \\
\hline Personas NO diagnosticadas & 307 & $(61,8 \%)$ & 240 & $(44,9 \%)$ & 547 & $(53,0 \%)$ \\
\hline Infecciosas y parasitarias & 5 & $(1,0 \%)$ & 9 & $(1,7 \%)$ & 14 & $(1,4 \%)$ \\
\hline Neoplasias & 1 & $(0,2 \%)$ & 1 & $(0,2 \%)$ & 2 & $(0,2 \%)$ \\
\hline Endocrino, metabólicas & 25 & $(5,0 \%)$ & 30 & $(5,6 \%)$ & 55 & $(5,3 \%)$ \\
\hline Sangre & -- & -- & 12 & $(2,2 \%)$ & 12 & $(1,2 \%)$ \\
\hline Trastornos mentales & 4 & $(0,8 \%)$ & 14 & $(2,6 \%)$ & 18 & $(1,7 \%)$ \\
\hline Sistema nervioso & 10 & $(2,0 \%)$ & 33 & $(6,2 \%)$ & 43 & $(4,2 \%)$ \\
\hline Circulatorias & 33 & $(6,6 \%)$ & 36 & $(6,7 \%)$ & 69 & $(6,7 \%)$ \\
\hline Respiratorias & 6 & $(1,2 \%)$ & 14 & $(2,6 \%)$ & 20 & $(1,9 \%)$ \\
\hline Digestivas & 27 & $(5,4 \%)$ & 19 & $(3,6 \%)$ & 46 & $(4,5 \%)$ \\
\hline Genitourinarias & 9 & $(1,8 \%)$ & 17 & $(3,2 \%)$ & 26 & $(2,5 \%)$ \\
\hline Embarazo, parto & -- & -- & 5 & $(0,9 \%)$ & 5 & $(0,5 \%)$ \\
\hline Piel & 5 & $(1,0 \%)$ & 8 & $(1,5 \%)$ & 13 & $(1,3 \%)$ \\
\hline Osteomuscular & 46 & $(9,3 \%)$ & 77 & $(14,4 \%)$ & 123 & $(11,9 \%)$ \\
\hline Mal definidas & 16 & $(3,2 \%)$ & 19 & $(3,6 \%)$ & 35 & $(3,4 \%)$ \\
\hline Accidentes, lesiones & 3 & $(0,6 \%)$ & 1 & $(0,2 \%)$ & 4 & $(0,4 \%)$ \\
\hline TOTAL & 497 & $(100,0 \%)$ & 535 & $(100,0 \%)$ & 1032 & $(100,0 \%)$ \\
\hline
\end{tabular}

Porcentaje del sexo encuestado entre paréntesis.

Fuente: Elaboración propia. 
de su salud, pues por un lado tienen una mayor esperanza de vida $y$ por otro presentan una peor situación socio-económica con respecto a sus compañeros.

Por último, en cuanto a la interacción de las variables relativas a la percepción de la salud, concluimos mediante un análisis discriminante sobre el índice general de salud que en cada uno de estos factores (morbilidad, asistencia sanitaria y conductas de riesgo), la mujer presenta una mayor vulnerabilidad. Por tanto, no es de extrañar que nos encontremos ante una situación circular: las conductas de búsqueda sanitaria se relacionan con una percepción negativa de la propia salud y con un mayor número de diagnósticos. Lo que no deja de sorprender en este proceso es que determinadas conductas de riesgo, como la inactividad o el consumo de tabaco, mediaticen, claramente, la evaluación del estado de salud.

\section{CONCLUSIONES E IMPLICACIONES POLÍTICAS}

No cabe duda de que, en nuestra sociedad, tanto hombres como mujeres difieren en sus comportamientos de salud y/o de riesgo. Por eso, la introducción de la variable sexo contribuye a una mejor comprensión de los procesos de salud-enfermedad (Doyal, 2000; Månsdotter, Lindholm and Lundberg, 2006). Hombres y mujeres difieren en sus molestias crónicas, mientras que para los primeros predominan los problemas de presión arterial alta, colesterol y alergias, para las segundas son más recurrentes los dolores de cabeza, los nervios o depresión.

Entre los principales resultados obtenidos destacamos que las mujeres tienden a valorar peor su estado de salud, presentando un mayor promedio de síntomas padecidos en los últimos 15 días. Sin embargo, aunque los hombres sufren menos complicaciones de salud, estas les incapacitan más. En general, las mujeres presentan una mayor vulnerabilidad, ratificándose de nuevo este dato mediante el análisis discriminante sobre el índice general de salud de la morbilidad, la asistencia sanitaria $y$ las conductas de riesgo.
Enmarcadas en estas coordenadas, es recomendable por un lado potenciar estilos de vida saludables, tal que por ejemplo promocionen el ejercicio físico entre las mujeres. Por otro lado, se debe continuar trabajando en la consecución de la igualdad entre hombres y mujeres. $\mathrm{Al}$ incrementarse su nivel educativo y renta disponible, pueden acceder a mejores y más adecuados bienes y servicios sanitarios, lo que sin duda repercutirá positivamente en su estado de salud.

Para conseguir los más altos niveles de salud, las políticas sanitarias deben reconocer que las mujeres y los hombres, debido a estas diferencias no sólo biológicas sino también socioeconómicas, no comparten las mismas necesidades, obstáculos y oportunidades. Requerimos para el cambio, un proceso técnico y político, que considere la cultura organizativa y la manera de pensar de la sociedad en su conjunto, así como en los objetivos, estructuras y distribución de los recursos. Por ello, es conveniente que los programas de salud pública incluyan una perspectiva definida $y$ transparente $y$ mejoren la capacidad estructural de coordinación entre los diferentes sectores implicados (Ministerio de Sanidad y Consumo, 2003).

Si bien es imprescindible elaborar políticas centradas en las mujeres, tampoco se debe olvidar diseñar estrategias propias para las personas mayores. Entre estas políticas destacaría el papel de las acciones que se emprendan con el fin de evitar la exclusión, como por ejemplo fomentando sus redes sociales (Daykin y Clarke, 2000; Mitchell and Schlesinger, 2006; Taylor y Hoening, 2006).

\section{BIBLIOGRAFÍA}

Braveman, P. y Tarimo, E. "Social inequalities in health within countries: not only an issue for affluent nations". Social Science and Medicine 54. 2002: 1621-1635.

Cais, J. y Castilla, G. El sector sanitario, sintesis $V$ Informe sociológico sobre la situación social en España. $1^{\text {a }}$ edición. Madrid: Documentación Social, 1995: 227-272. 
Canovos, G. C. Probabilidad y estadística. Aplicaciones y métodos. $1^{\mathrm{a}}$ edición. Madrid: McGraw-Hill, 1988.

Carpenter, M. "It's a small world: Mental policy under welfare capitalism since 1945". Sociology of health and illness 22. 5 . 2000: 602-620.

Carroll, S. "The personal is political". Women and Politics 9. 1989: 51-68.

Conrad, P y Schneider, J. Deviance and medicalization: From badness to sickness. $1^{\mathrm{a}}$ edición. Saint Louis: C.V. Mosby, 1980.

Cuesta, J. Historia de las mujeres en España. Siglo XX. $1^{\text {a }}$ edición. Madrid: Instituto de la Mujer, 2003.

Davis, A. y Ware, C. "Conceptualizations and measurements of health and patients for adults in the Health Insurance Study". J. Herrero, "Estresores sociales $y$ recursos sociales: El papel del apoyo social en el ajuste bio-psico-social". [Tesis doctoral]. Valencia, Universidad de Valencia. Facultad de Psicología, 1994.

Daykin, N. y Clarke, B. "They'll still get the bodily care. Discourses of care and relationships between nurses and health care assistants in the NHS". Sociology of health and illness 22.3. 2000: 349-363.

De León, V., Pelcastre, B. y Díaz, A. "Mujeres y Salud. Una metodología para su estudio. Chile: Facultad de Ciencias Sociales". [Revista electrónica Cinta de Moebio 9]. Universidad de Chile, 2000. En: <http:// www.moebio.uchile.cl/search.html>

De Miguel, J.M. La sociedad española 19961997. $1^{\text {a }}$ edición. Madrid: Universidad Complutense, 1997.

Estructura y cambio social en España. $1^{a}$ edición. Madrid: Alianza Editorial, 1998.
Dean, K. "El papel de la metodología en la creación de la información sesgada sobre salud de las mujeres. Vivir en salud, haciendo visibles las diferencias". Memorias del congreso sobre "Mujer, trabajo y salud". Barcelona, 1997. En: <http://nodo50.ix.apc. org/mujeresred/salud-caps.htm>

Deaton, A. "Health in an Age of Globalization". NBER Working Paper w10669, 2004.

Delgado, A. y López Fernández, L.A. "La Participación Comunitaria: Una revisión necesaria". Atención Primaria 32. 1992: 457- 459.

Dennis, R.; Williams, W.; Giangreco, M. y Cloninger, C. "Calidad de vida como contexto para la planificación y evaluación de servicios para personas con discapacidad". Siglo Cero 25. 155. 1994: 5-18.

Denny, K. "The effects of human capital on social capital: a cross-country analysis". Working Paper 03/16. The Institute for Fiscal Studies, 2003.

Diener, E. $y$ Larsen, R.J. "The experience of emotional well-being”. Diener, E. y Fujita, F. "Resources, personal strivings and subjective well-being: a nomothetic and idiographic approach". Journal of Personality and Social Psychology 68. 1995: 926-935.

Doyal, L. "Gender equity in health: debates and dilemmas". Social Science and Medicine 51. 2000: 931-939.

Driscoll, D., Halcoussis, D. y Svorny, S. "School district size and student performance". Economics of Education Review 22. 2003: 193-201.

Federación de Asociaciones de Vecinos. Zaragoza barrio a barrio: Casco Viejo, Casablanca. $1^{a}$ edición. Zaragoza: Ayuntamiento de Zaragoza, 1981.

Garcés, J. y Martínez, A. Necesidades colectivas y servicios sociales. Alicante: Actual, 1992. 
García Ferrando M. Socioestadistica: Introducción a la estadística en sociología. $4^{a}$ edición. Madrid: Alianza Universidad Textos, 1992.

García, M. y Soler, M. J. "Salud percibida y alteraciones psicosomáticas en la adolescencia temprana". Balaguer, I. Estilos de vida en la adolescencia. $1^{\mathrm{a}}$ edición. Valencia: Promolibro, 2002.

Gobierno de Aragón. II Plan de Acción Positiva para las mujeres en Aragón 1997-2000. $1^{\mathrm{a}}$ edición. Zaragoza: Gobierno de Aragón, 1998.

Gobierno de Navarra. ¿Cómo estamos salud? Encuesta de salud 1991. $1^{\text {a }}$ edición. Pamplona: Departamento de Salud, 1991.

Gómez, E. La salud y las mujeres en América Latina y el Caribe: viejos problemas y nuevos enfoques. $1^{\text {a }}$ edición. Agenda Salud 1/95 Isis Internacional. Washington: OPS, 1994: 4B.

Hellinger, F.J. and Encinosa, W. E. "Antiretroviral Therapy and Health Care Utilization: A Study of Privately Insured Men and Women with HIV Disease". Health Services Research 39.4. 2004: 949-967.

Månsdotter, A., Lindholm, L. and Lundberg, M. "Health, wealth and fairness based on gender. The support for ethical principles". Social Sciences and Medicine 62. 2006: 2327-2335.

Mateo Rivas M. J. y García Ferrando M. Estadística aplicada a las ciencias sociales. $1^{a}$ edición. Madrid: UNED, 1989.

Menéndez, E. Morir de alcohol. Saber y hegemonía médica. $1^{\mathrm{a}}$ edición. México: Alianza, 1990.

Ministerio de Sanidad y Consumo. La equidad de género en la salud. $1^{\mathrm{a}}$ edición. Madrid: Ministerio de Sanidad y Consumo, 2003.
Mitchell, S. and Schlesinger, M. "Managed Care and Gender Disparities in Problematic Health Care Experiences". Health Services Research 40. 5. 2006: 1489-1513.

Myers, D.G. $y$ Diener, E. "Who is happy?". Diener, E. y Fujita, F. "Resources, personal strivings and subjective well-being: a nomothetic and idiographic approach". Journal of Personality and Social Psychology 68. 1995: 926-935.

Sierra, R. Análisis estadístico multivariable: teoría y ejercicios. $1^{\text {a }}$ edición. Madrid: Paraninfo, 1994.

Serret, J. Manual de estadística universitaria. Inductiva. $1^{\text {a }}$ edición. Madrid: ESIC, 1995.

Sofaer, S. and Firminger, K. "Patients Perceptions of the Quality of Health Services". Annual Review of Public Health 26. 1. 2005: 513524.

Stevens, G.D., Seid, M., Mistry, R. and Halfon N. "Disparities in Primary Care for Vulnerable Children: The Influence of Multiple Risk Factors". Health Services Research 41.2. 2006: 507-531.

Taylor, D.H. and Hoening, H. "Access to health care services for the disable elderly". Health Services Research 41. 3. 2006: 743 758.

Thorson, A. and Johansson E. "Equality or equity in health care access: a qualitative study of doctors' explanations to a longer doctor's delay among female TB patients in Vietnam". Health Policy 68. 2004: 37-46.

Vanderzee, K.I.; Buunk, B.P. y Sanderman, R. "Social comparison as a mediator between health problems and subjective health evaluations". British Journal of Social Psychology 34. 1995: 53-65. 
